\title{
Multi-color, rotationally resolved photometry of asteroid 21 Lutetia from OSIRIS/Rosetta observations ${ }^{\star}$
}

\author{
P. L. Lamy ${ }^{1}$, G. Faury ${ }^{1}$, L. Jorda ${ }^{1}$, M. Kaasalainen ${ }^{2}$, and S. F. Hviid ${ }^{3}$ \\ ${ }^{1}$ Laboratoire d'Astrophysique de Marseille, UMR 6110 CNRS/Université de Provence, 38 rue Frédéric Joliot-Curie, \\ 13388 Marseille Cedex 13, France \\ e-mail: philippe.lamy@oamp.fr \\ 2 Tampere University of Technology, Finland \\ 3 Max-Planck-Institut für Sonnensystemforschung, Germany
}

Received 18 March 2010 / Accepted 19 June 2010

\begin{abstract}
Context. Asteroid 21 Lutetia is the second target of the Rosetta space mission. Extensive pre-encounter, space-, and ground-based observations are being performed to prepare for the flyby in July 2010.

Aims. The aim of this article is to accurately characterize the photometric properties of this asteroid over a broad spectral range from the ultraviolet to the near-infrared and to search for evidence of surface inhomogeneities.

Methods. The asteroid was imaged on 2 and 3 January 2007 with the Narrow Angle Camera (NAC) of the Optical, Spectroscopic, and Infrared Remote Imaging System (OSIRIS) during the cruise phase of the Rosetta spacecraft. The geometric conditions were such that the aspect angle was $44^{\circ}$ (i.e., mid-northern latitudes) and the phase angle $22.4^{\circ}$. Lutetia was continuously monitored over $14.3 \mathrm{~h}$, thus exceeding one rotational period and a half, with twelve filters whose spectral coverage extended from 271 to $986 \mathrm{~nm}$. An accurate photometric calibration was obtained from the observations of a solar analog star, 16 Cyg B.

Results. High-quality light curves in the $U, B, V, R$ and $I$ photometric bands were obtained. Once they were merged with previous light curves from over some 45 years, the sidereal period is accurately determined: $P_{\text {rot }}=8.168271 \pm 0.000002 \mathrm{~h}$. Color variations with rotational phase are marginally detected with the ultraviolet filter centered at $368 \mathrm{~nm}$ but are absent in the other visible and near-infrared filters. The albedo is directly determined from the observed maximum cross-section obtained from an elaborated shape model that results from a combination of adaptive-optics imaging and light curve inversion. Using current solutions for the phase function, we find geometric albedos $p_{V}=0.130 \pm 0.014$ when using the linear phase function and $p_{V}(H-G)=0.180 \pm 0.018$ when using the $(H-G)$ phase function, which incorporates the opposition effect. The spectral variation of the reflectance indicates a steady decrease with decreasing wavelength rather than a sharp fall-off.
\end{abstract}

Key words. minor planets, asteroids: general - minor planets, asteroids: individual: 21 Lutetia - techniques: photometric

\section{Introduction}

The Rosetta spacecraft was successfully launched on 2 March 2004 and is now on its way to rendezvous comet 67P/Churyumov-Gerasimenko in May 2014 at a heliocentric distance of 4.5 AU. During its journey to the comet, the spacecraft has already flown by asteroid 2867 Steins on 5 September 2008 (Keller et al. 2010). It will next flyby another main belt asteroid, 21 Lutetia, on 10 July 2010, at a velocity of $15 \mathrm{~km} \mathrm{~s}^{-1}$ and a closest approach of $3055 \mathrm{~km}$. As for all targets of space missions, an a-priori knowledge of these "terra incognita" is important for preparing and optimizing the operations of the spacecraft and its instruments so as to maximize the scientific return.

We have already performed a detailed characterization of asteroid 2867 Steins before its flyby in a series of four articles (Barruci et al. 2008; Jorda et al. 2008; Lamy et al. 2008a; Lamy et al. 2008b). Our solution for the shape and size was

\footnotetext{
* Photometric tables (Tables 4 to 8 ) are only available in electronic form at the CDS via anonymous ftp to

cdsarc.u-strasbg. fr $(130.79 .128 .5)$ or via

http: //cdsarc.u-strasbg.fr/viz-bin/qcat?]/A+A/521/A19
}

instrumental in preparing the observational sequences. It further served as a starting model for the three-dimensional reconstruction from the Rosetta images and helped complete the unseen part of the body (Jorda et al. 2010). The flyby was too short to address the question of the rotational state fortunately solved by our earlier pre-flyby observations (Lamy et al. 2008a). Finally, our determination of the geometric albedo obtained by combining visible and infrared measurements (Lamy et al. 2008b) has been shown to be remarkably accurate by the in situ photometric analysis performed by Spjuth et al. (2010). We now turn our attention to the second target asteroid, 21 Lutetia, and present multi-color, rotationally resolved observations obtained with the OSIRIS narrow angle camera (NAC) onboard the Rosetta spacecraft, and discuss its photometric properties (color, albedo).

At about $100 \mathrm{~km}$ in diameter, 21 Lutetia is a large main belt asteroid discovered on 15 November 1852 by H.M.S. Goldschmidt (an amateur astronomer) at the Paris Observatory. Although its investigation has picked up momentum in recent years in anticipation of the upcoming Rosetta flyby, its nature remains controversial. Its early classification as an M-type asteroid based on eight-color photometry and a large IRAS albedo (Barucci et al. 1987; Tholen 1989) has been questioned, and 
Table 1. Summary of the observations of 21 Lutetia obtained with the OSIRIS-NAC using 12 filters.

\begin{tabular}{cccccc}
\hline \hline Code & Name & $\bar{\lambda}$ & $\Delta \lambda$ & $\tau$ & $N_{\text {obs }}$ \\
\hline F16 & FFP-UVNEAR_UV & 367.6 & 36.7 & 600 & 26 \\
F24 & FFP-VISBLUE & 479.9 & 74.0 & 90 & 26 \\
F23 & FFP-VISGREEN & 536.4 & 64.5 & 90 & 26 \\
F22 & FFP-VISORANGE & 648.6 & 85.2 & 90 & 26 \\
F28 & FFP-VISRED & 742.4 & 63.2 & 90 & 26 \\
F51 & ORTHOFFP-IR & 804.7 & 40.6 & 240 & 26 \\
F41 & NEAR_IRFFP-IR & 880.1 & 63.6 & 210 & 26 \\
F15 & FFP-UVFAR_UV & 270.7 & 47.0 & 600 & 2 \\
F27 & FFP-VISHYDRA & 701.2 & 22.0 & 300 & 2 \\
F61 & OLIVINE/FFP-IR & 931.7 & 34.7 & 600 & 2 \\
F71 & IR/FFP-IR & 986.1 & 34.5 & 600 & 2 \\
F58 & ORTHO/RED & 790.1 & 26.3 & 600 & 2 \\
\hline
\end{tabular}

Notes. $\bar{\lambda}=$ mean wavelength $(\mathrm{nm}), \Delta \lambda=$ equivalent width $(\mathrm{nm}), \tau=$ exposure time (s), $N_{\text {obs }}=$ number of images.

the closest meteorite analogues to its surface composition appear to be particular types of carbonaceous chondrites such as CO3, CV3, and CH (e.g., Barucci et al. 2008).

Lutetia was recently classified in the Xc subclass (DeMeo et al. 2009); among its very few members, 97 Klotho presents spectral (Vernazza et al. 2009) and polarization (Belskaya et al. 2010) properties similar to those of Lutetia.

The purpose of the present investigation is to take advantage of a space telescope, namely the OSIRIS narrow angle camera (NAC) onboard the Rosetta spacecraft, to monitor Lutetia without the usual limitations of ground-based observations (diurnal cycle, atmospheric transmission). We obtained a homogeneous set of complete, well-sampled light curves whose spectral coverage extends from 368 to $880 \mathrm{~nm}$ and additional data extending the spectral domain from 271 to $986 \mathrm{~nm}$, which allowed us to address the questions of the spectral reflectance, of possible color variations across the surface, and of the albedo.

\section{Observations}

The narrow-angle camera (NAC) is the high-resolution camera of the OSIRIS imaging system (Keller et al. 2007) onboard ROSETTA. The NAC is a three-mirror anastigmat telescope with an unobstructed aperture of $90 \mathrm{~mm}$ and a focal length of $717 \mathrm{~mm}(f / D=8)$. In addition to its original optical concept for a planetary camera, the NAC implements a novel all-siliconcarbide mechanical design where the mirrors and the telescope structure are made of the same material SiC (Dohlen et al. 1996; Calvel et al. 1999). It is equipped with a dual filter wheel and an E2V 42-40 2048 2 pixel backlit, back-thinned CCD. The pixel size of $13.5 \mu \mathrm{m}$ corresponds to 3.88 arcsec. The NAC offers an excellent point source sensitivity. The optical point spread function has a $F W H M$ of $8.3 \operatorname{arcsec}(2.15$ pixels) and is adequately sampled by the CCD detector (see Fig. 1 of Jorda et al. 2008).

The observations started on 2.917 January 2007 UT and ended on 3.513 UT, thus covering an overall time span of $14.3 \mathrm{~h}$. The geometric parameters varied only slightly over this interval: the heliocentric distance changed from 2.5772 to $2.5763 \mathrm{AU}$, the distance to the Rosetta spacecraft from 1.6406 to $1.6433 \mathrm{AU}$, and the solar phase angle increased from 22.26 to $22.48^{\circ}$. Lutetia was observed with 12 different filters whose central wavelengths range from 271 to $986 \mathrm{mn}$ (Table 1 and Fig. 1). They include both broad band filters for photometric characterization and narrow
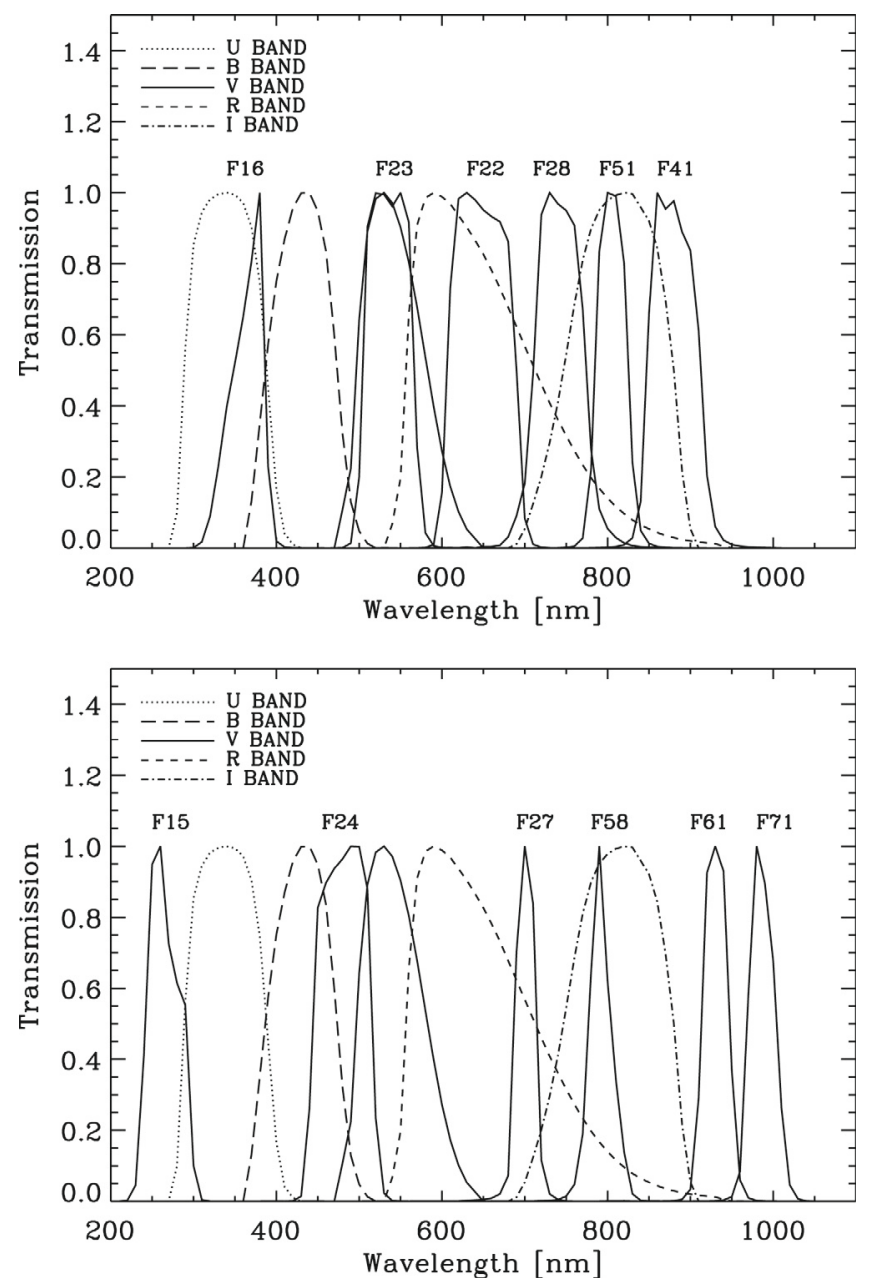

Fig. 1. Spectral transmissions of the 12 OSIRIS-NAC filters normalized to their peak values. Standard Johnson-Kron-Cousins $U B V R I$ bands are overplotted using different dashed/dotted lines for comparison.

band filters for mineralogic characterization. The observational program started with 24 identical sequences taking place every $30 \mathrm{mn}$. Each sequence included seven images successively acquired with filters F16, F24, F23, F22, F28, F51, and F41, and it lasted $23.5 \mathrm{mn}$. Subsequently, five filters were added to the above list, F15, F27, F61, F71, and F58, and this new sequence which lasted $45 \mathrm{mn}$ was repeated twice without interruption. The overall program is summarized in Table 1 and a total of 192 images acquired. Each image corresponds to a window of $256 \times 256$ pixels centered on the expected position of Lutetia. The spacecraft tracking the moving target was very accurate with an error within a single exposure smaller than the PSF of the camera. Figure 2 displays a set of 12 images taken with the 12 filters, each one an enlargement centered on the asteroid.

\section{Data reduction}

\subsection{Image analysis}

The data were reduced with the OSIRIS standard calibration pipeline that implements the following operations:

- the CCD is read out using a dual 14 bit analog-to-digital converter (ADC). DN values created by the "upper" ADC (DN values $\geq 2^{14}$ ) get an additional offset added by the readout electronics. This offset is subtracted; 


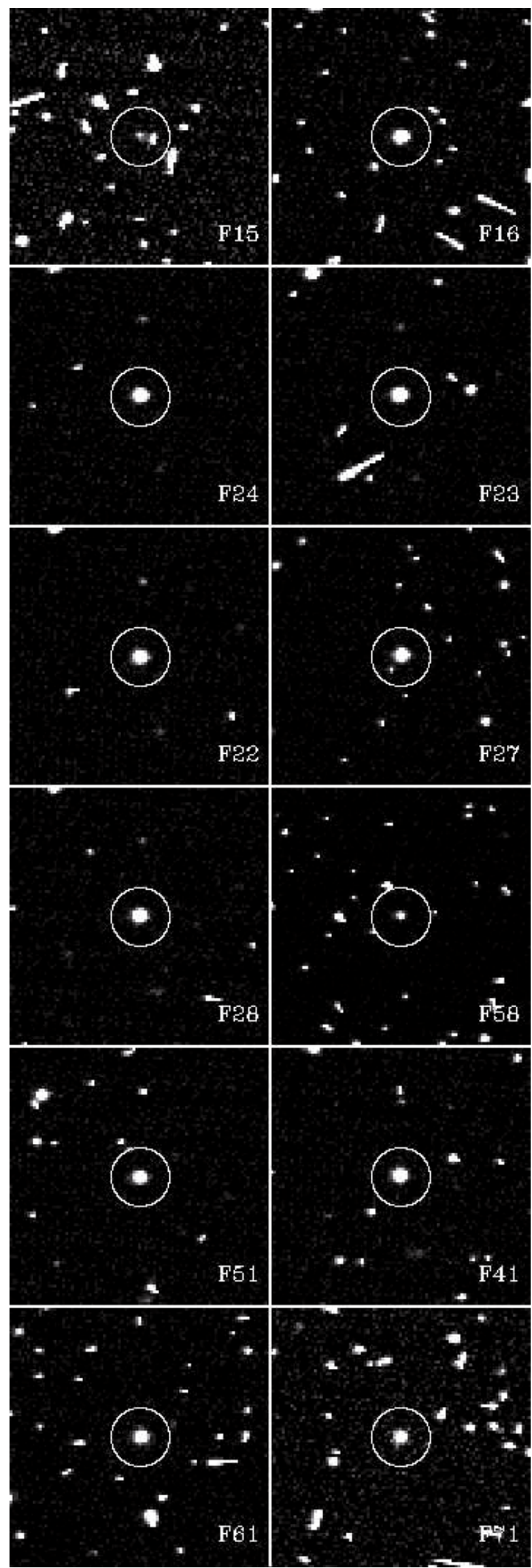

Fig. 2. Enlarged subframe images of asteroid 21 Lutetia obtained with the 12 OSIRIS-NAC filters. Each one is centered on Lutetia highlighted by a circle. Note the excellent tracking by the spacecraft.

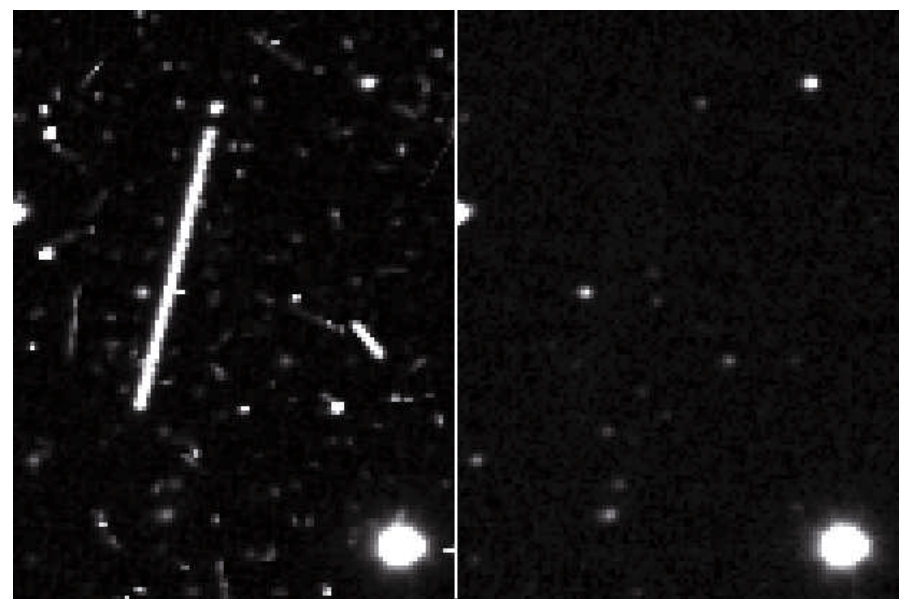

Fig. 3. Left panel: image of the sum of the individual 26 co-registered images obtained with the F22 filter where the asteroid appears as a bright linear track. Right panel: image of the reference background for the F22 filter.

- coherent noise produced by the power converter is filtered out;

- the bias value estimated from bias exposures taken immediately after the Lutetia observations is subtracted;

- the images are divided by the flat field relevant to the same filter.

The photometric analysis of the images follows the method developed by Jorda et al. (2008) for the previously observed asteroid 2867 Steins and insures that the flux measurements are properly corrected from contributions of any neighboring stars. For each of the 7 sets of 26 images, the first step consisted in constructing a reference background image from the 26 images accurately co-registered on the star positions at subpixel level. In practise, we used a single reference star free of any artifacts (saturation, impacts of cosmic rays). Figure 3 displays the sum of these 26 co-registered images obtained with the F22 filter, where the asteroid appears as a bright linear track. The background image itself was constructed by taking the median value of the 26 values at each pixel, thus efficiently removing the asteroid track and all artifacts.

The subpixel positions of the asteroid were first determined on each individual original images, and then transformed to instantaneous positions on the background image. Circular aperture photometry was performed using the IDL procedure "APER" from the ASTRON library, separately for the 26 original images of the asteroid and for the background image at the corresponding positions. As for asteroid Steins, we selected an aperture radius of 2 pixels as the best compromise between recording the bulk of the flux of the point spread function and limiting the spurious contributions from neighboring stars. This procedure was not really appropriate to the two sets of images obtained with the additional five filters and we implemented "SExtractor", an advanced procedure developed for reducing large-scale galaxy-survey data, but also adapted to the photometry of crowded fields (http://www.astromatic.net/ software/sextractor). 


\subsection{Uncertainty}

We followed the method implemented by Jorda et al. (2008) for the photometry of Steins and expressed the $1 \sigma$ error of the data as

$s=\sqrt{\frac{B}{g}+\left(\frac{\mathfrak{R}}{g}\right)^{2}+(f B)^{2}}$

where $B$ is the observed signal in $\mathrm{DN}, g=3$ electrons $\mathrm{DN}^{-1}$ is the gain, $\mathfrak{R}=7$ electrons is the readout noise, and $f=0.01$ expresses the flat field noise as a fraction of the signal.

\subsection{Absolute calibration}

The solar spectral analog 16 Cyg B was observed with the 12 filters as part of the in-flight calibration of the NAC, and photometric measurements were likewise performed using the same aperture, i.e., a radius of 2 pixels. In parallel, synthetic photometry was performed to obtain a consistency check and to verify the absence of any red leaks in the ultraviolet filters, the usual point of concern for these filters. We used the solar spectrum from Colina et al. (1996) and the throughput of the 12 channels of the NAC calculated from the calibrated transmissions of all optical components and the calibrated quantum efficiency of the detector. For all channels, the direct and synthetic photometries of 16 Cyg B agree at the $~ 1 \%$ level.

The 16 Cyg B photometry enabled us to determine the zero points $(\mathrm{ZP})$ of the transformation between the standard $U B V R I$ magnitudes of the Johnson-Kron-Cousins system and the instrumental magnitudes measured with the NAC filters. We associated the standard bands to the neighboring filters as follows: the $U$ band to the F16 filter, the $B$ band to F16 and F24, the $V$ band to F23 and F24 (note that the F23 filter approximately matches the $V$ band), the $R$ band to F22 and F28, and the $I$ band to F28, $\mathrm{F} 41$, and F51.

These zero points were corrected for the effect of the color of asteroid Lutetia. This was performed according to the procedure developed by Jorda et al. (2008) for Steins. In summary, the corrections of the zero points are given by equations of the type:

$\Delta_{Z P}=-2.5 \log \frac{\int_{V} S_{\mathrm{Lut}}(\lambda) \mathrm{d} \lambda \times \int_{\mathrm{NAC}} F_{\odot}(\lambda) \mathrm{d} \lambda}{\int_{V} F_{\odot}(\lambda) \mathrm{d} \lambda \times \int_{\mathrm{NAC}} S_{\mathrm{Lut}}(\lambda) \mathrm{d} \lambda}$

where $F_{\odot}(\lambda)$ is the solar spectrum, $S_{\text {Lut }}(\lambda)$ is the Lutetia spectrum, and the integrals extend over the standard photometric bands (the $V$ band in the above example) and one of the NAC bandpass. The Lutetia spectrum is expressed as

$S_{\text {Lut }}(\lambda)=R_{\text {Lut }}(\lambda) F_{\odot}(\lambda)$

where $R_{\text {Lut }}(\lambda)$ is the (relative) spectral reflectivity of Lutetia.

The solar spectrum $F_{\odot}(\lambda)$ is taken from Colina et al. (1996). The reflectivity of Lutetia was generated from the 320-1000 nm spectrum obtained by Barucci et al. (2005) on 16 November 2004. We extrapolated the decreasing trend down to $200 \mathrm{~nm}$ (Fig. 4) consistent with the widespread behavior of the reflectivity of asteroids in the ultra violet; this extrapolation is not critical since it concerns a second-order correction of the zero points.

The spectral profiles of the standard bands were taken from Landolt (1992).

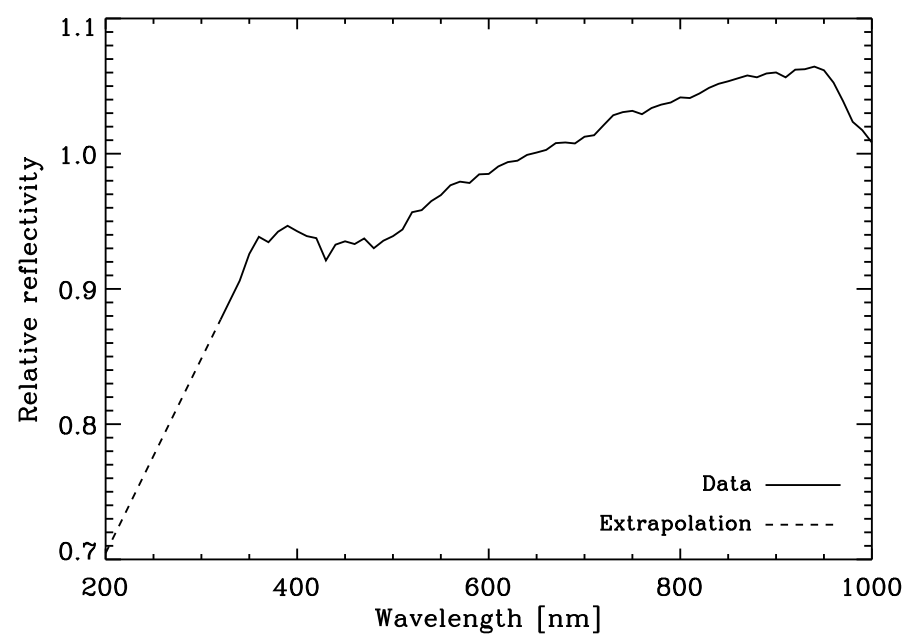

Fig. 4. Relative spectral reflectivity of asteroid 21 Lutetia used for calculating the color corrections applied to the zero points of the photometric transformation formulae.

\section{Results}

\subsection{Multi-color light curves of asteroid 21 Lutetia}

Figure 5 displays the light curves of asteroids 21 Lutetia for the $U B V R I$ bands where the reduced magnitudes $m\left(1,1, \alpha=22.4^{\circ}\right)$ are plotted as functions of time. As explained in the above section, either one $(U)$, two $(B V R)$, or three $(I)$ filters were associated to the standard photometric bands as indicated in parentheses. In the case of multiple light curves, i.e., two for the $B V R$ bands and three for the $I$ band, the agreements are excellent with dispersions always lying within the $1 \sigma$ error bars.

The measured magnitudes $m\left(1,1, \alpha=22.4^{\circ}\right)$ for the five photometric bands are reported in Tables 4 to 8 (available at the CDS). The $V$ light curve has a mean value of $V=8.371 \pm 0.002$ and an amplitude of $0.121 \pm 0.023 \mathrm{mag}$. Our data, together with recent ground-based light curves, were merged with the data set used by Torppa et al. (2003) to extend the overall temporal coverage to 48 years and therefore to refine the determination of the sidereal period: $P_{\text {rot }}=8.168271 \pm 0.000002 \mathrm{~h}$ ( see the above reference for details of the method and error estimation).

Our rotationally-folded $V$ light curve is displayed in Fig. 6. Although we are clearly seeing the cross-section of an elongated body, the light curve does not exhibit the canonical doublepeaked behavior of a simple ellipsoid in rotation around its short axis and viewed from its equatorial plane. The geometry of our observations can be understood by considering two recently determined shape models, the triaxial ellipsoid derived by Drummond et al. (2010) from adaptive-optics (AO) imaging acquired at the Keck telescope and a more elaborated model that we constructed by combining $\mathrm{AO}$ imaging acquired at the VLT telescope (Carry et al. 2010) and inversion of a large set of 50 light curves, including the present ones. The direction of the rotational axis of this model is defined by its ecliptic coordinates $\lambda=52^{\circ}$ and $\beta=-6^{\circ}$ with an uncertainty of $3^{\circ}$. It implies an aspect angle (defined as the angle between the spin vector and the asteroid-observer vector) for our observations of $44^{\circ}$, i.e., an intermediate view between equatorial and polar thus partly explaining our "irregular" light curve. Figure 7 displays four views for each model as seen by the OSIRIS/NAC during the observational campaign at four different phase angles. We then compared our $V$ light curve with the one calculated from the elaborated shape model assuming a Lommel-Seeliger scattering 


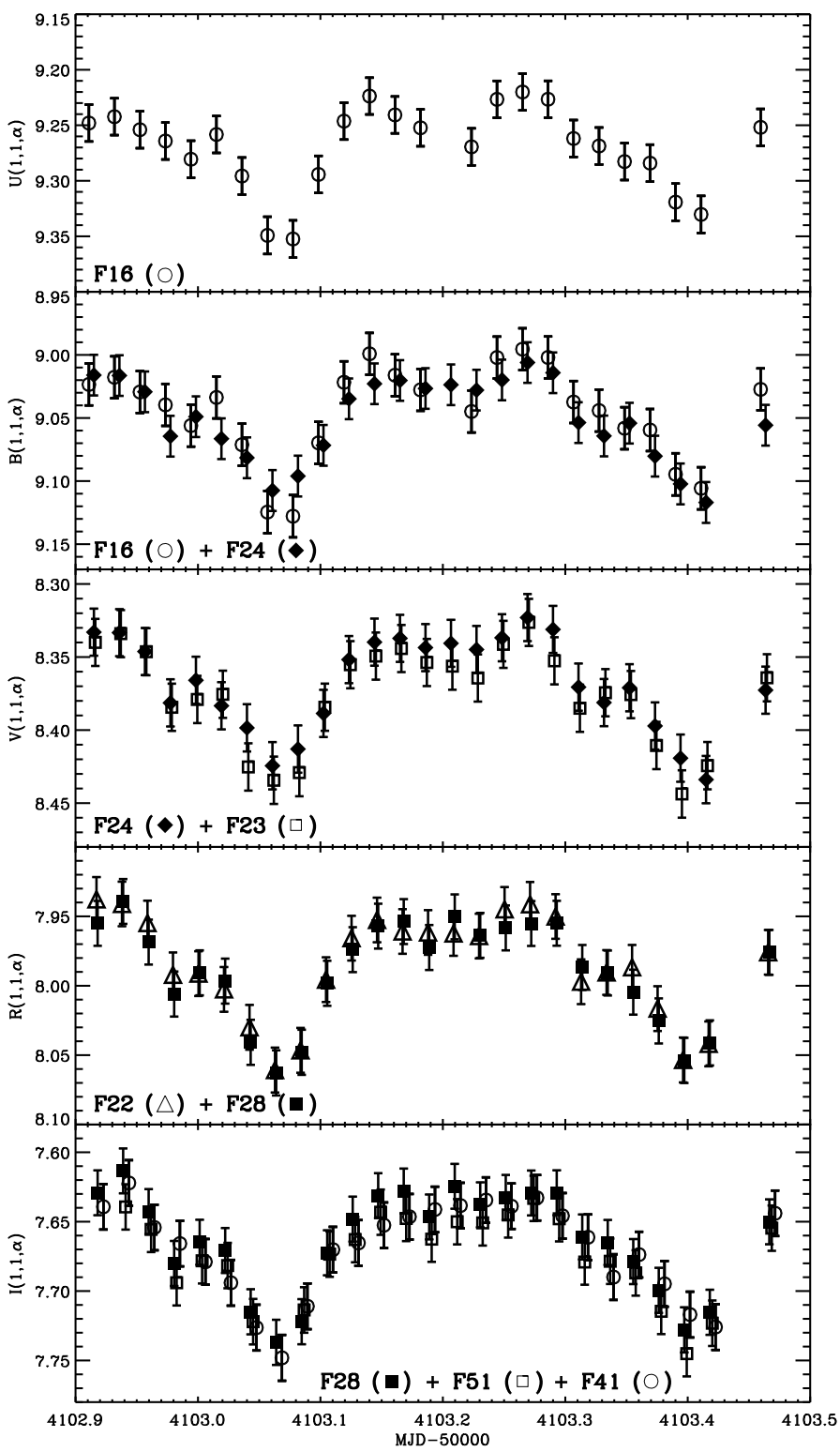

Fig. 5. Light curves of asteroid 21 Lutetia. The $U B V R I$ reduced magnitudes are plotted as function of the MJD.

law (Fig. 6). Having in mind the inherent difficulties of a shape model to accurately match a large set of light curves, the actual fit is quite satisfactory with $\mathrm{rms}$ deviation of $\sim 0.003 \mathrm{mag}$. The scale of the graph tends to exaggerate all features; for instance, the "secondary maximum" at a rotational phase of $\sim 0.25$ is only a $\sim 0.02$ mag (i.e., $2 \sigma$ ) bump over the model. The deviations are quite typical of this kind of fit, and the "missing maximum" and slight deviations at the other maxima could result from shape features not representable at the resolution scale of the model, or subtle variations of albedo/scattering law.

The question of the rotationally resolved photometry of Lutetia is best tackled by directly considering the ratios of the various fluxes measured in the different filters. We selected the F22 band as a reference since it recorded the largest signals (largest signal-over-noise ratios) and since these measurements were roughly performed near the midpoint of each sequence. Incidentally, during a sequence lasting $23.5 \mathrm{mn}$, the asteroid rotates by $17^{\circ}$ which is acceptable for this kind of analysis. We calculated the ratios of the F22 fluxes to those of the six other

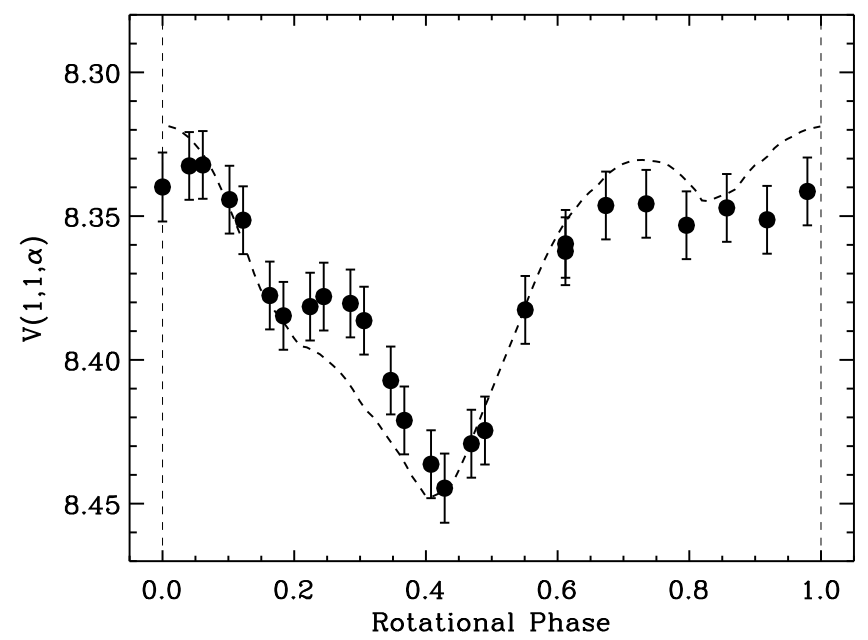

Fig. 6. Folded $V$ band light curve of asteroid 21 Lutetia. The $V(1,1, \alpha=$ $22.4^{\circ}$ ) magnitudes are plotted as function of the rotational phase (filled circles), together with the calculated light curve from the elaborated shape model (dashed line).

filters and display them folded with the rotational period of Lutetia thus representing color variations as a function of rotational phase (Fig. 8). The regions of overlap allow to assess the consistency of the measurements as the flux ratios shifted by one rotation always agree within the $1 \sigma$ uncertainty. With the exception of the F22/F16 ratio, all other ratios pertaining to the 480-880 $\mathrm{nm}$ spectral range remained constant, the observed fluctuations never departing from the mean values by more than $1 \sigma$. The F22/F16 ratio which involves the ultra violet filter centered at $368 \mathrm{~nm}$ exhibits more conspicuous variations than the other ratios, slightly exceeding the $1 \sigma$ bar over the mean value. To further ascertain these variations, we introduced the ratio F41/F16 which therefore involves the largest wavelength range available in our data. Variations similar to that of the F22/F16 ratio are observed but still barely exceeding $2 \sigma$ for the largest ones. These conclusions can be quantified by considering the upper limits of the variations (i.e., the ratio of the maximum to minimum values) together with their uncertainities as given in each panel of Fig. 8. These upper limits are typically 3\%, reaching $5 \%$ for the $\mathrm{F} 22 / \mathrm{F} 16$ ratio and up to $6.5 \%$ for the $\mathrm{F} 41 / \mathrm{F} 16$ ratio, with however $1 \sigma$ uncertainties of $\sim 2.5 \%$. We may tentatively conclude for marginal evidence of compositional variations of the surface of Lutetia, which could then involve minerals having signature(s) in the ultraviolet such as electronic charge transfer bands. When comparing with reports of variations in the shape of visible spectra (the so-called spectral slope) with rotational phase, the aspects of the observations must be taken into account: south polar view for Prokof'eva-Mikhailovskaya et al. (2007), for Belskaya et al. (2010) and for Perna et al. (2010), equatorial view for Busarev (2008). Note that the above aspects have been recalculated on the basis of our solution for the pole direction and that they may differ from those quoted by the respective authors based on different solutions. Variations in the shape of nearinfrared spectra have also been reported by Nedelcu et al. (2007), here again at equatorial aspect. Our conclusion of the absence of spectral variations in the visible and marginal evidence in the ultraviolet at mid-northern latitudes therefore do not contradict the above reports and further indicate marked compositional variegation across the surface of Lutetia. Forthcoming spatiallyresolved measurements expected from the Rosetta instruments will hopefully shed new light on this question. 

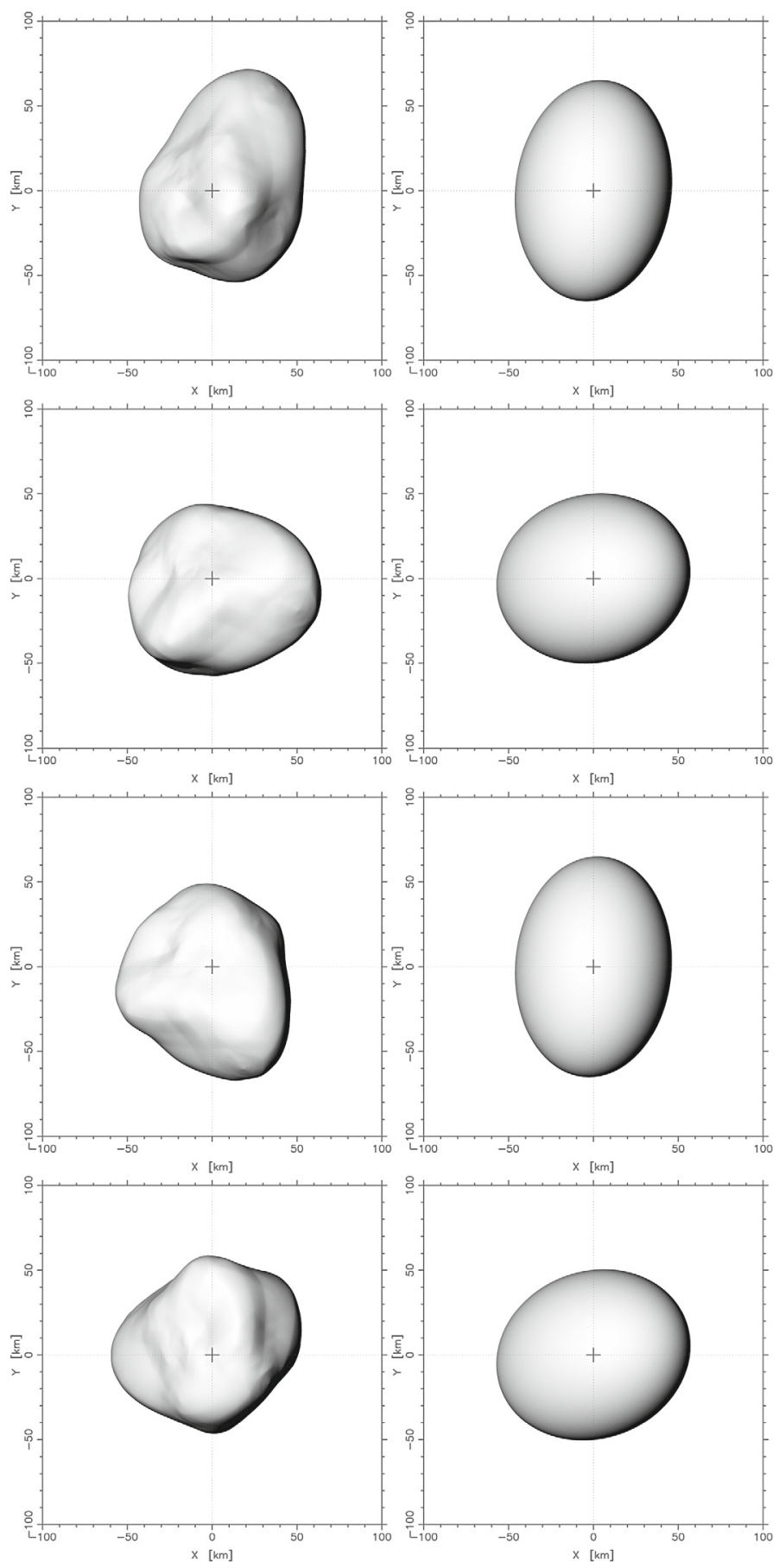

Fig. 7. Three-dimensional views of asteroid 21 Lutetia as seen by the OSIRIS/NAC at four different rotational phases corresponding to the extrema of the light curve. Left column: the present shape model, right column: ellipsoid model from Drummond et al. (2010).

\subsection{The albedo of asteroid 21 Lutetia}

To determine the albedo of Lutetia, we considered the elaborated shape model introduced above and exploited the fact that the AO images yield the absolute size of the body. We calculated the maximum cross-section that would have been seen by the NAC at zero phase angle, $1.05 \times 10^{10} \mathrm{~m}^{2}$. Our magnitude corresponding to the maximum of the $\mathrm{V}$ light curves must be corrected to zero phase angle and we relied on the phase functions constructed by Belskaya et al. (2010) from a large set of data (Fig. 9). Their H-G solution (Bowell et al. 1989) at near

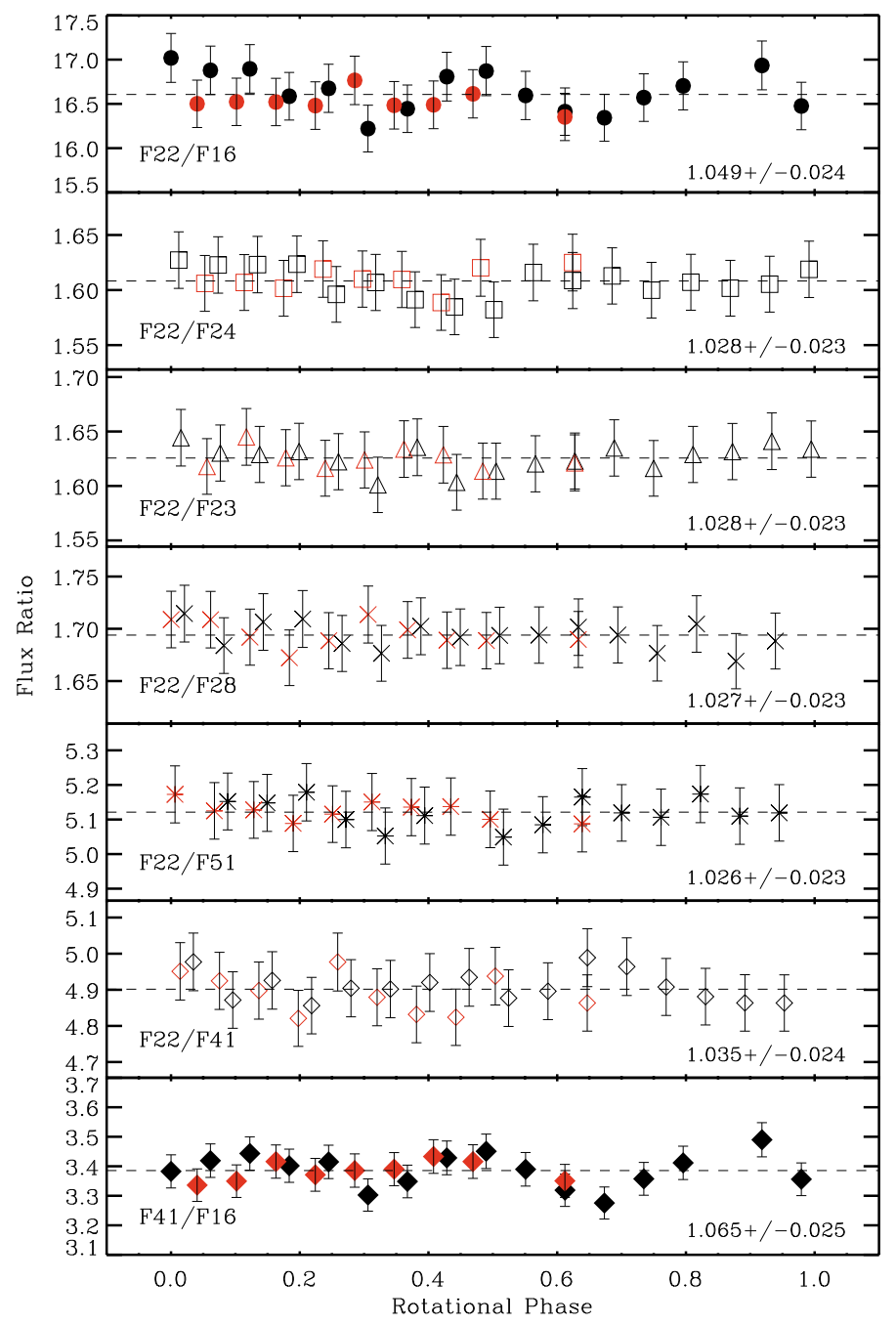

Fig. 8. Ratios of the fluxes obtained with the reference F22 filter to those obtained with the other filters and the F41/F16 ratio (bottom panel). The fluxes are plotted versus rotational phase and the black/red symbols distinguish the two successive rotations of Lutetia. The dashed lines correspond to the mean values of the respective data sets. Upper limits of the variations are given in each panel.

polar aspect angles is characterized by $G=0.12 \pm 0.01$ and leads to $V(1,1,0)=7.210 \pm 0.023$. Their linear phase function, which removes the opposition effect has a phase coefficient $\beta=$ $0.034 \pm 0.001 \mathrm{mag} \mathrm{deg}^{-1}$, and leads to $V(1,1,0)=7.560 \pm 0.026$ The above uncertainties on $V(1,1,0)$ were obtained by propagating the errors affecting our measurement and the $G$ and $\beta$ parameters. It should be noted that the coincidence between our results and those of Belskaya et al. (2010, their Fig. 3) is totally fortuitous because the respective observations were obtained at different aspect angles. The standard formula relating magnitudes to cross-sections, originally devised by Russell (1916) for asteroids observed at large phase angles and conveniently reformulated by Jewitt (1991), allowed us to calculate the geometric albedo $p$ in a given photometric band. Following our analysis of asteroid 2867 Steins (Jorda et al. 2008), we considered two cases:

1. the linear phase function yields an albedo representative of the global photometric properties of the asteroid $p_{V}=$ $0.130 \pm 0.014$, consistent with the definition of the geometric albedo, which relies on the comparison with a Lambertian diffuser (Hanner et al. 1981); 


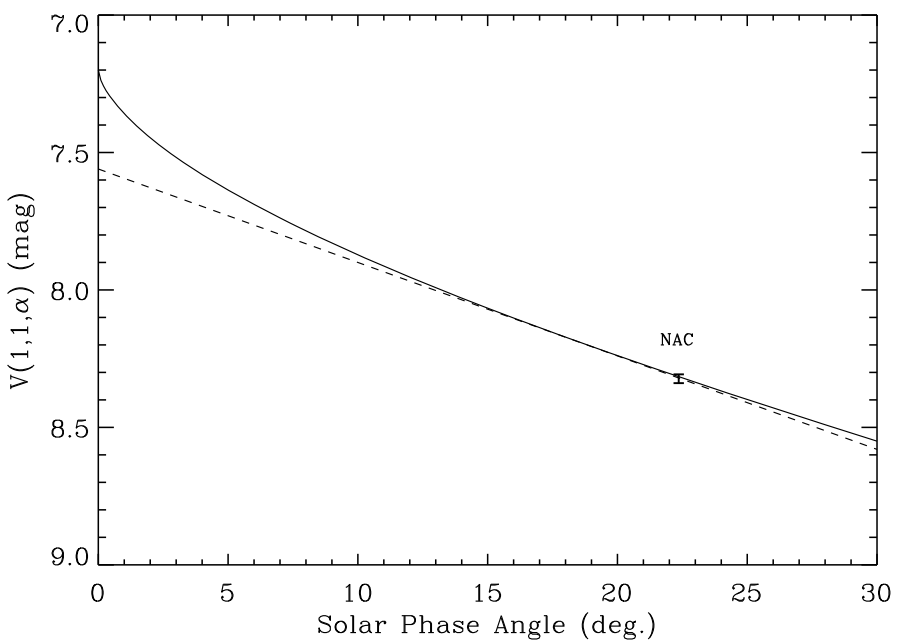

Fig. 9. Phase function $V(1,1, \alpha)$ of asteroid 21 Lutetia. Our result from the NAC observations (maximum of the $V$ light curves) is displayed with its error bar. The phase functions fitted to our data point are from Belskaya et al. (2010): ( $H-G)$ function (solid line) and linear function (dashed line).

Table 2. Determinations of the geometric albedo of 21 Lutetia.

\begin{tabular}{lcc}
\hline \hline Author & Method & Albedo \\
\hline Tedesco \& Veeder (1992) & Thermal & $0.221 \pm 0.02$ \\
Zellner \& Gradie (1976) & Pol. (h) & 0.10 \\
Lupishko \& Mohamed (1996) & Pol. & 0.10 \\
Magri et al. (1999) & Radar & $0.19 \pm 0.07$ \\
Mueller et al. (2006) & Thermal & $0.208 \pm 0.025$ \\
Gil-Hutton (2007) & Pol. $\left(P_{\min }\right)$ & $0.11 \pm 0.01$ \\
Gil-Hutton (2007) & Pol. (h) & $0.20 \pm 0.04$ \\
Carvano et al. (2008) & Thermal & $0.13 \pm 0.03$ \\
Shepard et al. (2008) & Radio & $0.24 \pm 0.07$ \\
Weaver et al. (2010) & Vis + 3D model & $0.16 \pm 0.02$ \\
Belskaya et al. (2010) & Pol. (h) & $0.13 / 0.16 \pm 0.02 *$ \\
This work & Vis + 3D model & $0.18 \pm 0.02$ \\
\hline
\end{tabular}

Notes. ${ }^{*} p_{V}=0.13 \pm 0.02$ when using the calibration based on albedos of meteorites (Zellner \& Gradie 1976) and $p_{V}=0.16 \pm 0.02$ when using the calibration based on IRAS albedos (Cellino et al. 1999).

2. the $(H-G)$ phase function incorporates the opposition effect so that the albedo is artificially enhanced: $p_{V}(H-G)=$ $0.180 \pm 0.018$. While this albedo is of standard use for classifying asteroids, we emphasize that it is irrelevant when solving for the thermal emission of the asteroid.

The error bars on the albedo were determined assuming a $10 \%$ uncertainty on the cross-sections which represents the dominating contribution.

Various methods have been applied to determine the albedo of Lutetia, and the results are summarized in Table 2. The radar albedo values range from 0.19 (Magri et al. 1999) to 0.24 (Shepard et al. 2008), both values affected by the same large uncertainty of \pm 0.07 ; consequently, the radar albedo is not strongly constrained and may be as low as 0.12 at the $1 \sigma$ level. The thermal albedo values range from 0.13 (Carvano et al. 2008) to 0.21-0.22 (Tedesco \& Veeder 1992; Mueller et al. 2006) with error bars of 0.02 to 0.03 . The determination of Carvano et al. (2008) is identical to our value obtained without the opposition surge that is relevant to thermal modeling as argued above (see also Lamy et al. 2008b). We show in a separate article that this value is further compatible with extended, rotationally-resolved thermal measurements of Lutetia performed with the Spitzer space telescope (Lamy et al. 2010). The value of $0.22 \pm 0.02$ by Tedesco \& Veeder (1992) was determined at zero phase angle using absolute magnitudes $\mathrm{H}$, so it should be compared to our value $p_{V}=0.18 \pm 0.02$, leading to an agreement within the error bars. A closer look at the article by Mueller et al. (2006) reveals that their result is an average of 5 determinations obtained with different models and different data sets and that the individual determinations range from 0.19 to 0.24 with errors ranging from 0.04 to 0.06 making a couple of them fully compatible with our two determinations. All authors who performed polarization measurements of Lutetia emphasize the peculiar behavior of the negative branch of polarization so that applying the empirical calibrations relating albedo to either $P_{\min }$ or $h$ remains problematic. However, the most recent results by Belskaya et al. (2010) are very encouraging.

- Their value $p_{V}=0.13 \pm 0.02$ is obtained by applying the empirical h-albedo relationship calibrated with albedos of meteorites measured in laboratory at a phase angle of $5^{\circ}$ (Zellner \& Gradie 1976) i.e., almost without opposition effect. It is identical to our determination obtained under the same assumption.

- Their value $p_{V}=0.16 \pm 0.02$ is obtained by applying the calibration based on IRAS albedos determined at zero phase angle using the absolute magnitude $\mathrm{H}$ (Cellino et al. 1999). It should be compared to our determination using the HG phase function $p_{V}=0.18 \pm 0.02$, and both determinations agree within their error bars.

Weaver et al. (2010) used a method similar to ours, combining photometry performed with the Hubble space telescope with the ellipsoid shape model of Drummond et al. (2010). They obtained $p_{V}=0.16$ with an uncertainty dominated by the shape model and therefore close to ours, that is, $\sim 0.02$. Consequently, our results agree within the error bars.

In conclusion, it appears that the reality of the discrepancies between various determination of the albedo of Lutetia has been exaggerated. Taking the various uncertainties in the measurements into account, in the models (and assumed parameters), in the empirical calibrations, and of paramount importance, distinguishing between albedos with and without opposition effect, most if not all reported values can be reconciled. We are confident that our determinations based on accurate space photometry and on the most advanced shape model, and which further agree with the recent results of Belskaya et al. (2010) and of Weaver et al. (2010), reflect the real properties of the surface of Lutetia.

Several groups (e.g., Magri et al. 1999; Shepard et al. 2008; Belskaya et al. 2010) have concluded that an albedo in the range 0.13-0.18 excludes a metallic surface composition and is consistent with particular types of carbonaceous chondrites and enstatite chondrites. On the other hand, Belskaya \& Shevchenko (2000) derive a linear relationship between the phase coefficient (or slope) of the linear part (denoted "b") and the logarithm of the visible albedo $p_{V}$. With a slope $b=0.034 \pm 0.001 \mathrm{mag} / \mathrm{deg}$ and $p_{V}(H-G)=0.180 \pm 0.018$, Lutetia lies close to this linear relationship and clearly stands outside the group of C-type objects and well within the M-type group. This is one piece of evidence of the puzzling nature of Lutetia. 
Table 3. Color indices of asteroid 21 Lutetia.

\begin{tabular}{|c|c|c|c|c|}
\hline Filters & $(U-B)$ & $(B-V)$ & $(V-R)$ & $(R-I)$ \\
\hline$(F 16-F 24)$ & $0.22 \pm 0.02$ & $0.68 \pm 0.02$ & & \\
\hline$(F 16-F 23)$ & $0.21 \pm 0.02$ & $0.67 \pm 0.02$ & & \\
\hline$(F 24-F 23)$ & & $0.68 \pm 0.02$ & & \\
\hline$(F 24-F 22)$ & & & $0.38 \pm 0.02$ & \\
\hline$(F 24-F 28)$ & & & $0.38 \pm 0.02$ & \\
\hline$(F 23-F 22)$ & & & $0.39 \pm 0.02$ & \\
\hline$(F 23-F 28)$ & & & $0.39 \pm 0.02$ & \\
\hline$(F 22-F 28)$ & & & & $0.32 \pm 0.02$ \\
\hline$(F 22-F 51)$ & & & & $0.31 \pm 0.02$ \\
\hline$(F 22-F 41)$ & & & & $0.32 \pm 0.02$ \\
\hline Mean values & $0.22 \pm 0.01$ & $0.68 \pm 0.01$ & $0.39 \pm 0.01$ & $0.32 \pm 0.01$ \\
\hline Sun & 0.19 & 0.65 & 0.35 & 0.28 \\
\hline PDS & $0.19 \pm 0.02$ & $0.69 \pm 0.03$ & & \\
\hline$Z+75$ & $0.19 \pm 0.03$ & $0.72 \pm 0.01$ & & \\
\hline $\mathrm{L}+83$ & & $0.67 \pm 0.02$ & & \\
\hline $\mathrm{D}+00$ & & $0.68 \pm 0.02$ & & \\
\hline $\mathrm{B}+10$ & & $0.65 \pm 0.01$ & $0.42 \pm 0.01$ & $0.34 \pm 0.01$ \\
\hline
\end{tabular}

Notes. The first block of data gives the individual color indices calculated from the various combinations of NAC filters used to derive the $U B V R I$ magnitudes. The second block gives the corresponding mean values of the indices and those of the Sun for comparison. The third block gives results found in the literature: PDS = Planetary Data System; Z+75 = Zellner et al. (1975); L+83 = Lupishko et al. (1983); $\mathrm{D}+00=$ Denchev (2000); B+10 = Belskaya et al. (2010).

\subsection{The color of asteroid 21 Lutetia}

We derived the color indices of Lutetia from the mean values of the $U B V R I$ magnitudes and display our results separately for the various filter combinations in Table 3 , showing the excellent consistency of these different determinations, hence demonstrating the quality of the photometric analysis. We finally averaged the corresponding determinations and obtained $(U-$ $B)=0.22 \pm 0.01,(B-V)=0.68 \pm 0.01,(V-R)=0.39 \pm 0.01$, and $(R-I)=0.32 \pm 0.01$.

It is interesting to recall the implications of these color indices, in particular those involving the $U B V$ bands, for classifying Lutetia (e.g., Zellner et al. 1975). In the color-color diagram $(U-V)$ versus $(B-V)$, Lutetia strikingly lies well inside the taxonomy group $M$ and completely outside the $C$ group. While it is not possible to classify an object only with photometric colors, this result adds more evidence of the puzzling nature of this asteroid.

\subsection{Spectral reflectance and albedo of asteroid 21 Lutetia}

Lutetia was observed with 12 different filters covering the spectral range 271 to $986 \mathrm{~nm}$ in two consecutive identical sequences,

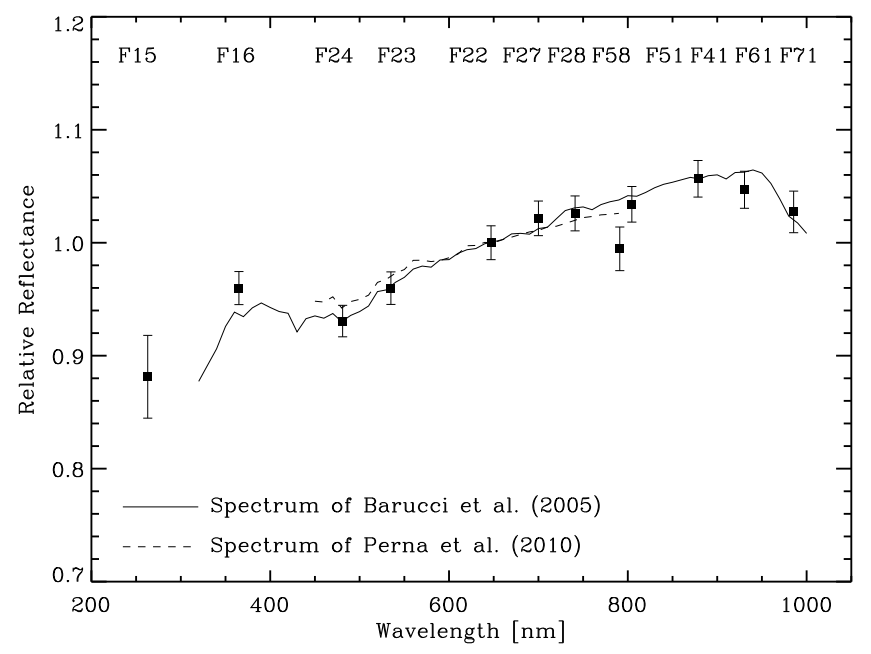

Fig. 10. Relative spectral reflectance of asteroid 21 Lutetia. Our results (data points) are compared with the spectra of Barucci et al. (2005) and of Perna et al. (2010).

each lasting $45 \mathrm{mn}$. As the overall duration reached $1.5 \mathrm{~h}$, a significant fraction of the rotational period, and as the observations further corresponded to a rising branch of the light curve, it was not possible to average the pairs of measurements and readily construct the spectral reflectance. In addition, the first of the two observations taken with the F15 filter was unfortunately unusable as the image of the asteroid significantly departed from the point spread function of the NAC (a faint cosmic ray impact was suspected) leading to an incoherent flux. A linear interpolation was constructed from each pair of flux measurements and sampled at the time of the second F15 observation, thus insuring the simultaneity of the multispectral data.

The solar spectral analog 16 Cyg B was observed with the same filters so that the relative reflectance of Lutetia was obtained simply by taking the ratios of the corresponding fluxes. Our results, together with the spectrum of Barucci et al. (2005) obtained on 16 November 2004 and that of Perna et al. (2010) obtained on 28.1 November 2008, are displayed in Fig. 10. The overall agreement is excellent. We note a slight anomaly of the measurement with the F58 filter as the suggested absorption band is not present in the spectra. It may result from a calibration problem, but it disappears at the $2 \sigma$ level.

We next generated the spectral geometric albedo of Lutetia by scaling the reflectance data to $p_{V}(H-G)=0.18$ at the central wavelength of the $V$ band $(540 \mathrm{~nm})$ as shown in Fig. 11. We superimposed:

- the original IUE data reproduced from Table II of Roettger \& Buratti (1994);

- the IUE data point at $267 \mathrm{~nm}$ recently corrected by Weaver et al. (2010) using a different phase function and a new effective diameter;

- the HST results from Weaver et al. (2010).

For those data, we introduced a (systematic) uncertainty of $10 \%$ to account for the error on the asteroid cross-section in addition to the quoted photometric uncertainties. As already discussed above, the NAC and HST albedo values in the visible agree within their $1 \sigma$ error bars, which amount to $\sim 0.02$. The situation is more problematic in the ultraviolet. The HST data suggest a sharp drop in the spectral range 300-330 nm, while our data together with the corrected IUE data point at $267 \mathrm{~nm}$ support a more gentle decrease as the wavelength decreases. New 


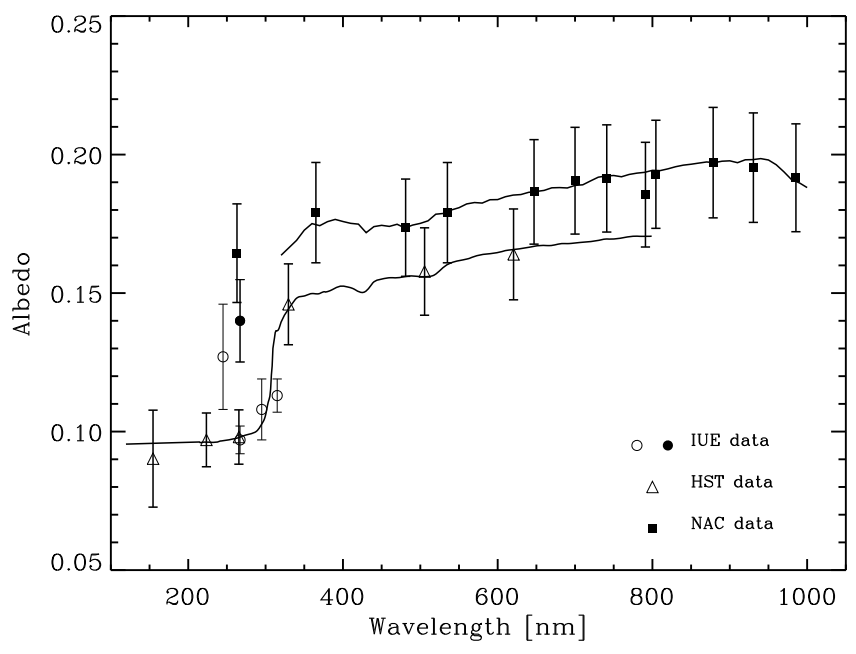

Fig. 11. Spectral albedo of asteroid 21 Lutetia. The IUE data points correspond to the original data from Roettger \& Buratti (1994) and appear as open circles, while the one at $267 \mathrm{~nm}$ has been corrected by Weaver et al. (2010) and appears as a filled circle. The curve going through the NAC data points corresponds to the spectrum of Barucci et al. (2005), while the curve going through the HST data points corresponds to the best estimate by Weaver et al. (2010).

observational data, in particular from the Rosetta instruments, are needed to clarify these contradictory results and to establish the true albedo of Lutetia in the ultraviolet.

\section{Summary and conclusions}

We have presented an in-depth photometric analysis of the observations of the Rosetta mission flyby target asteroid 21 Lutetia obtained with the OSIRIS narrow angle camera onboard the Rosetta spacecraft. Our analysis includes an accurate absolute calibration using a solar analog star. Our main findings are summarized below.

1. The photometric data obtained with 7 different filters ranging from 368 to $880 \mathrm{~nm}$ and regularly sampling 1.65 rotational period yielded consistent light curves in the $U B V R I$ bands of the Johnson-Kron-Cousins photometric system. Merging with a large set of light curves obtained over some 45 years, the sidereal period was accurately determined as $P_{\text {rot }}=8.168271 \pm 0.000002 \mathrm{~h}$.

2. Color variations with rotational phase were marginally detected with the ultraviolet filter centered at $368 \mathrm{~nm}$ but were absent in the other visible and near-infrared filters, a result that strictly applies to mid-northern latitutes. It therefore does not contradict variations reported for south polar and equatorial views, and it further suggests marked compositional variegation across the surface of Lutetia.

3. The spectral variation of the reflectance in the domain 271-986 nm obtained thanks to including 5 additional filters indicates a progressive decrease with decreasing wavelength rather than a sharp fall-off as reported by Weaver et al. (2010). This would, to some extent, alleviate the difficulty pointed out by these authors in finding a reasonable analog among measured samples.

4. The albedo was directly determined from the observed maximum cross-section obtained from an elaborated shape model. We found geometric albedos $p_{V}=0.130 \pm 0.014$ when using the linear phase function and $p_{V}(H-G)=$ $0.180 \pm 0.018$ when using the $(H-G)$ phase function that incorporates the opposition effect. These values are much higher than those typical of common C-type asteroids $(\sim 0.06)$ and are best compatible with either some types of carbonaceous chondrites (prominently enstatite chondrites and marginally $\mathrm{CO}$ ) or M-type asteroids.

5. The $(U-B)$ and $(B-V)$ color indices show that Lutetia lies well inside the taxonomy group $M$ and completely outside the $C$ group.

In addition, the mass estimates of $2.57 \pm 0.24 \times 10^{18} \mathrm{~kg}$ by Baer et al. (2008) and of $2.06 \pm 0.60 \times 10^{18} \mathrm{~kg}$ by Fienga et al. (2009), combined with a volume of $5 \pm 1 \times 10^{14} \mathrm{~m}^{3}$ resulting from our shape model, leads to bulk densities of $5.1 \pm 1.1$ and $4.1 \pm 1.5 \mathrm{~g} \mathrm{~cm}^{-3}$, respectively, that is an overall range from 2.6 to $6.1 \mathrm{~g} \mathrm{~cm}^{-3}$. These are extraordinarily large values for asteroids based on current data. In the present view where asteroids are thought to have large macroporosity, this would require that the component material has even a higher density, henceforth a substantial metallic content. An alternative interpretation excludes significant porosity and probably a rubble-pile internal structure for Lutetia. In this context, we can directly compare the above result with the grain densities of meteorites: $\sim 2.3 \mathrm{~g} \mathrm{~cm}^{-3}$ for $\mathrm{CI}$ and $\mathrm{CM}, \sim 3.5 \mathrm{~g} \mathrm{~cm}^{-3}$ for $\mathrm{CO}$ and $\mathrm{CV}, 3.6$ to $3.7 \mathrm{~g} \mathrm{~cm}^{-3}$ for $\mathrm{CH}$, and 4.4 to $4.6 \mathrm{~g} \mathrm{~cm}^{-3}$ for stony-iron meteorites (Britt $\&$ Consolmagno 2003; Consolmagno et al. 2008; Macke et al. 2009). Excluding the CI and CM, they are all compatible with the present uncertainty range of the density of Lutetia.

As summarized by Belskaya et al. (2010), several lines of independent evidence suggest a similarity of the properties of the surface of Lutetia with those of particular types of carbonaceous chondrites (e.g., $\mathrm{CO} 3, \mathrm{CV} 3, \mathrm{CH}$ ): i) minor features in its visible spectra indicative of aqueous alteration (Lazzarin et al. 2004, 2009; Busarev 2004); ii) the $3 \mu \mathrm{m}$ feature indicative of hydrated silicates (Rivkin et al. 2000; Birlan et al. 2006); iii) its overall thermal 5.2-32 $\mu \mathrm{m}$ emissivity spectrum and the presence of Christiansen, Reststrahlen and Transparency features (Barucci et al. 2008); iv) its particular polarization properties as already mentioned in Sect. 4.2. In addition, Barucci et al. (2008) have emphasized the similarity of the visible and near-infrared spectra of Lutetia with those of the CO3 (e.g., Ornans) or CV3 (e.g., Vigarano) type meteorites, contrary to that of Odessa, an iron meteorite that contains some silicate inclusions, while Vernazza et al. (2009) favor enstatite chondrites.

However, if forthcoming measurements lead to a large bulk density, then a more complex internal structure would have to be considered for Lutetia; for instance, the (oversimplified) picture of a monolithic, prominently metallic body with a thick regolith layer with small grain size that underwent some aqueous alteration could partly reconcile the current observational evidence. In particular, this would explain the very low thermal inertia (Mueller et al. 2006; Carvano et al. 2008; Lamy et al. 2010), but the composition of the regolith would have to be consistent with the constraints listed above.

Further requirements would come from the near and far ultraviolet spectra.

Ultimately, the problem probably lies in understanding the peculiar physical properties of Lutetia and how such an object can form, either primordially or by subsequent evolution. Rather than speculating further on these questions, we prefer to wait for the vast crop of data expected from the forthcoming flyby that hopefully will shed some new light on these puzzling properties.

Acknowledgements. OSIRIS was built by a consortium of the MaxPlanck-Institut für Sonnensystemforschung, Lindau, Germany, the Laboratoire d'Astrophysique de Marseille, France, the Centro Interdipartimentale Studi e 
Attivita' Spaziali, University of Padova, Italy, the Instituto de Astrofisica de Andalucia, Granada, Spain, the Research and Scientific Support Department of the European Space Agency (ESA/ESTEC), Noordwijk, The Netherlands, the Instituto Nacional de Tecnica Aerospacial, Madrid, Spain, the Institut für Datentechnik und Kommunikationsnetze der Technischen Universitat, Braunschweig and the Department of Astronomy and Space Physics of Uppsala University, Sweden. The support of the national funding agencies DLR, CNES, ASI, MEC, NASA, and SNSB is gratefully acknowledged. We thank the personnel of the Rosetta Science Operations Center at ESA/ESTEC and of the Rosetta Mission Operations Center at ESA/ESOC for their prompt and efficient scheduling of the observations. We are grateful to I. Belskaya, D. Perna and H. Weaver for making their results available to us in advance of publication, to S. Croft for providing us with the color indices of asteroids, and to P. Vernazza for an enlightning discussion. This research made use of JPL's online ephemeris generator (horizons).

\section{References}

Baer, J., Milani, A., Chesley, S., \& Matson, R. D. 2008, Bul. Am. Astron. Soc. 40, 493

Barucci, M. A., Capria, M. T., Coradini, A., \& Fulchignoni, M. 1987, Icarus, 72, 304

Barucci, M. A., Fulchignoni, M., Fornasier, S., et al. 2005, A\&A, 430, 313

Barucci, M. A., Fornasier, S., Dotto, E., et al. 2008, A\&A, 477, 665

Belskaya, I. N., \& Shevchenko, V. G. 2000, Icarus, 147, 94

Belskaya, I. N., Fornasier, S., Krugly, et al. 2010, A\&A, 515, A29

Birlan, M., Vernazza, P., Fulchignoni, M., et al. 2006, A\&A, 454, 677

Bohlin, R. C., Dickinson, M. E., \& Calzetti, D. 2001, AJ, 122, 2118

Bowell, E., Hapke, B., Domingue, D. et al., 1989, in Asteroids II (Univ. of Arizona Press), 524

Britt, D. T., \& Consolmagno, G. J. 2003, Meteorit. Planet. Sci., 38, 1161

Busarev, V. V. 2004, Lunar and Planetary Institute Science Conference Abstracts, 35,1026

Busarev, V. V. 2008, LPI Contributions, 1405, 8010

Calvel, B., Castel, D., Lamy, P. L., Dohlen, K., \& Bougoin, M. 1999, Advanced Telescope Design. Proc. SPIE, 3785, 56

Carry, B., Kaasalainen, M., Leyrat, C., et al. 2010, A\&A, in press [arXiv: 1005.5356]

Carvano, J. M., Barucci, M. A., Delbó, M., et al. 2008, A\&A, 479, 241

Cellino, A., Hutton, R. G., Tedesco, E. F., di Martino, M., \& Brunini, A. 1999, Icarus, 138, 129

Colina, L., Bohlin, R. C., \& Castelli, F. 1996, AJ, 112, 307

Consolmagno, G. J., Britt, D. T., \& Macke, R. J. 2008, Lunar and Planetary Institute Science Conference Abstracts, 39, 1582

DeMeo, F. E., Binzel, R. P., Slivan, S. M., \& Bus, S. J. 2009, Icarus, 202, 160

Denchev, P. 2000, Planet. Space Sci., 48, 987

Dohlen, K., Saisse, M., Claeysen, G., Lamy, P. L., \& Boit, J.-L. 1996, Optic. Engi. 35, 1150
Dollfus, A. \& Geake, J. E. 1975, Proceedings of the 6th Lunar Science Conference, Houston, Texas, March 17-21, 3, 2749

Drummond, J. D., Conrad, A., Merline, W., et al. 2010, A\&A, in press [arXiv: 1005.5353$]$

Feierberg, M. A., Witteborn, F. C., \& Lebofsky, L. A. 1983, Icarus, 56, 393 Fienga, A., Laskar, J., Morley, T., et al. 2009, A\&A, 507, 1675

Gil-Hutton, R. 2007, A\&A, 464, 1127

Hanner, M. S., Giese, R. H., Weiss, K., \& Zerull, R. 1981, A\&A, 104, 42

Jewitt, D. 1991, Comets in the post-Halley era, 167, IAU Colloq, 11619

Jorda, L., Lamy, P. L., Faury, G., et al. 2008, A\&A, 487, 1171

Jorda, L., Lamy, P. L., Groussin, O., et al. 2010, Icarus, to be submitted

Keller, H. U., Barbieri, C., Lamy. P. L., et al. 2007 Space Sci. Rev., 128, 26

Keller, H. U., Barbieri, C., Koschny, D., et al. 2010, Science, 327, 190

Lamy, P. L., Toth, I., Weaver, H. A., et al. 2006, A\&A, 458, 669

Lamy, P. L., Kaasalainen, M., Lowry, S. et al. 2008a, A\&A, 487, 1179

Lamy, P. L., Jorda, L., Fornasier, S., et al. 2008b, A\&A, 487, 1187

Lamy, P. L., Groussin, O., Fornasier, S., et al., 2010, A\&A, 516, A74

Landolt, A. U. 1992, AJ, 104, 340

Lazzarin, M., Marchi, S., Magrin, S., Barbieri, \& C.Lazzarin, M. 2004, A\&A, 425, L25

Lazzarin, M., Marchi, S., Moroz, L. V., \& Magrin, S. 2009, A\&A, 498, 307

Lee, W., \& Wdowiak, T. J. 1993, ApJ, 417, L49

Lupishko, D. F., \& Mohamed, R. A. 1996, Icarus, 119, 209

Lupishko, D. F., Belskaya, I. N., \& Tupieva, F. A. 1983, Pis'ma Astron. Zh., 9, 691

Macke, R. J., Britt, D. T., \& Consolmagno, G. J. 2009, Lunar and Planetary Institute Science Conference Abstracts, 40, 1598

Magri, C., Ostro, S. J., Rosema, K. D., et al. 1999, Icarus, 140, 379

Merline, W., Drummond, J., Conrad, A., et al. 2010, A\&A, to be submitted

Mueller, M., Harris, A. W., Bus, S. J., et al. 2006, A\&A, 447, 1153

Nedelcu, D. A., Birlan, M., Vernazza, et al. 2007, A\&A, 470, 1157

Perna, D., Dotto, E., Lazzarin, M., et al. 2010, A\&A, 513, L4

Prokof'eva-Mikhailovskaya, V. V., Batrakov, Y. V., Bochkov, V. V., Busarev,

V. V., \& Karachkina, L. G. 2007, Solar Syst. Res., 41, 307

Rivkin, A. S., Howell, E. S., Lebofsky, L. A., Clark, B. E., \& Britt, D. T. 2000, Icarus, 145, 351

Roettger, E. E., \& Buratti, B. J. 1994, Icarus, 112, 496

Russell, H. N. 1916, ApJ, 43, 173

Shepard, M. K.; Clark, B. E., Nolan, M. C., et al. 2008, Icarus, 195, 184

Spjuth, S., Jorda, L., Lamy, P. L., et al. 2010, Icarus, submitted

Tedesco \& Veeder, 1992, in The IRAS Minor planet Survey, Tech. Rep. PL-TR-

92-2049, Phillips Laboratory, Hanscom Air Force Base, Massachusetts

Tholen, D. J. 1989, in Asteroids II (Univ. of Arizona Press), 1139

Torppa, J., Kaasalainen, M., Michalowski, T., et al. 2003, Icarus, 164, 346

Vernazza, P., Brunetto, R., Binzel, R. P., et al. 2009, Icarus, 202, 477

Weaver, H. A., Feldman, P. D., Merline, W. J., et al. 2010, A\&A, 518, A4

Zellner, B., \& Gradie, J. 1976, AJ, 81, 262

Zellner, B., Wisniewski, W. Z., Andersson, L., \& Bowell, E. 1975, AJ, 80, 986 